\title{
Performance of a discrete Linear Quadratic Gaussian controller with a realistic noise model for adaptive optics
}

\author{
Mikhail V. Konnik and James S. Welsh
}

\begin{abstract}
Modern adaptive optics systems represent a significant challenge for automatic control. The output disturbance must be compensated in real time despite significant measurement noise and system delays. This paper presents the formulation of a discrete Linear Quadratic Gaussian controller with a realistic model of the measurement noise. The cases of high, medium, and low measurement noise are considered. The influence of the measurement noise on the discrete Linear Quadratic Gaussian controller in adaptive optics systems is discussed. The analysis of numerical stability of the discrete controller is provided.
\end{abstract}

Index Terms - robotics, optimal control, numerical simulations

\section{INTRODUCTION}

A DAPTIVE Optics systems aim to compensate for the influence of the Earth's atmosphere on observations of space from ground-based telescopes. The continuous movement of the atmosphere introduces inhomogeneity in the air refractive index, which leads to blurring of astronomical images acquired by ground-based telescopes. Adaptive optics can compensate for the turbulence in real-time using wavefront sensors (WFS) and deformable mirrors (DM). This is a closedloop operation, which is performed by a controller that generates commands for the DM using noisy and delayed measurements from a wavefront sensor.

Usually the controllers are formulated as simple PI controllers [1], where the gain can be adjusted for each actuator [2]. More advanced controllers such as Linear Quadratic Gaussian (LQG) controllers have been designed and simulated for modal wavefront correction $[3,4]$ in classical adaptive optics. The experimental demonstration of LQG controllers in adaptive optics was provided in [5]. The on-line parameter adjustment for LQG control was also proposed[6]. The experimental validation of optimal control[5, 7] was shown to outperform the classical control.

Linear Quadratic Gaussian (LQG) control for adaptive op-

Manuscript received 31 August, 2012, Revised 27 September, 2012.

Mikhail V. Konnik is with the School of Electrical Engineering and Computer Science, The University of Newcastle, Callaghan 2308, Australia; e-mail: mail@mvkonnik.

James S. Welsh is Faculty of Engineering and Built Environment, The University of Newcastle, Callaghan 2308, Australia; e-mail: James.Welsh@newcastle.edu.au tics systems is designed in simplified (no actuator dynamics) and unrealistic (no measurement noise) assumptions. This paper provides the results of numerical simulations to assess the performance of an LQG controller using a realistic measurement noise model. We use a comprehensive model of the CCD photosensor[8] to obtain the corresponding noise levels for the wavefront sensor in the adaptive optics system model. The correspondence between the photosensor noise in the WFS and the measurement noise is difficult to elaborate analytically. Various cases of photosensor ADC quantisation, signal and noise levels are considered in the numerical simulations provided in this paper. The numerical stability of the formulated LQR control was analysed. An alternative formulation of the optimal control with better numerical properties is discussed.

The paper is organized as follows. The dynamic model of an adaptive optics is formulated in Section Error! Reference source not found., including models of a wavefront sensor, a deformable mirror, and a model of the output disturbance. The LQG control problem is formulated in Section Error! Reference source not found. Then the initial parameters of the numerical simulations are presented in Section Error! Reference source not found.. The equivalent measurement noise derivation is described in Section Error! Reference source not found. Results on simulation of the measurement noise influence on the performance of a discrete LQG controller are provided in Section Error! Reference source not found. Discussion of simulation results is provided in Section Error! Reference source not found.. Finally, an important discussion of numerical stability of the optimal control is presented in Section Error! Reference source not found.

\section{THE FORMULATION OF THE CONTROL PROBLEM IN ADAP- TIVE OPTICS}

From the control standpoint, the adaptive optics system is a Linear Time Invariant (LTI) multi-input-multi-output (MIMO) system that consists of a sensor (wavefront sensor), a controller, and a corrector (deformable mirror) for the real-time sensing and compensation of an output disturbance (atmospheric turbulence). The system is a subject to both an output disturbance (atmospheric turbulence that must be corrected) and measurement noise from the CCD or CMOS photosensor inside the WFS. The aim of the controller is to track zero refer- 
ence (a flat wavefront) in the presence of output disturbances and significant measurement noise.

\section{A. The model of the wavefront sensor}

The dynamics of the wavefront sensor (WFS) are characterised by the integration time $T_{f}$ of the photosensor. The output of the sensor is the average of the signal from $t$ to $t+T_{f}$. The delay due to data processing is denoted by $\tau_{w f s}$. Therefore, the transfer function of the WFS can be represented in the Laplace domain as:

$$
G_{W F S}=\frac{\left(1-e^{T_{f} s}\right) e^{\tau_{w f s}}}{T_{f} S}
$$

The discrete data of the wavefront are contaminated by measurement noise from the WFS, which is described in detail in the Subsection A..

\section{B. The model of the Deformable mirror}

The dynamics of the continuous-faceplate deformable mirror (DM) with piezoelectric actuators can be divided into two parts: the dynamics of the surface, and the dynamics of the actuators. In this paper, only the dynamics of the actuators are considered. The coupling between actuators and the surface dynamics is neglected. The dynamics of the actuators[9] in a $\mathrm{DM}$ is given by:

$$
G_{D M}(s)=\frac{1}{s / a+1}
$$

where the parameter $a$ depends on the specific equipment used in the adaptive optics system.

\section{The model of the output disturbance}

The output disturbance in this system is due to atmospheric turbulence, which is essentially a wavefront phase distortion. The wavefront can be thought of as a surface of light rays coming from a distant star. If there is no atmospheric turbulence, the wavefront surface will be flat, which means that all the rays have passed the same distance. The atmospheric turbulence produces inhomogeneity in the air refractive index that leads to different path lengths for different light rays. Hence the wavefront will be non-flat, which causes the blur on astronomical images. The goal of the controller in the adaptive optics system is to counteract the non-flat (turbulent) wavefront by bending the DM in the opposite direction.

The atmospheric turbulence can be represented by a discrete-time state space model driven by a white noise. A state space model of the atmospheric turbulence (output disturbance) is given by:

$$
\begin{aligned}
& x_{a t m}[k+1]=A_{a t m} x_{a t m}[k]+B_{a t m} \boldsymbol{\xi}_{a t m}[k] \\
& y_{a t m}[k]=C_{a t m} x_{a t m}[k]
\end{aligned}
$$

where $x_{\text {atm }}[k]$ is the disturbance state and $\xi_{a t m}[k]$ is a zero mean, unit variance white noise process.

It was shown[10] that an autoregressive, AR(1), model provides limited performance due to the nature of the approximate model. On the other hand, an AR(5) model leads to a loss of performance due to increased sensitivity to variations of temporal characteristics of the atmospheric turbulence. The $\operatorname{AR}(2)$ model provides a reasonable agreement with the Kolmogorov model [11] in good seeing conditions and is used in this paper.

\section{The complete model of the Adaptive Optics system}

The complete model of the system consists of WFS dynamics, actuators of the DM, and a non-integer time delay of the wavefront sensor. In order to account for the non-integer time delay, we divide the delay into an integer part $n$ and fractional part $m \in[0,1)$. Therefore, the complete system delay $\tau_{d}$ will be represented as: $\tau_{d}=(n-m) \cdot T_{f}$. Because $n$ is an integer, the term $\exp \left[-n T_{f} s\right]$ will be transformed in the Zdomain as $z^{-n}$. The residual fractional delay $\exp \left[m T_{f} s\right]$ will be absorbed into the coefficients of the discretized model [12].

The discretized equivalent of the continuous system can be represented as a transfer function:

$$
\begin{aligned}
& G_{s y s}(z)=\frac{z \beta+\alpha \beta}{z^{n+2}-z^{n+1} e^{-\alpha T_{f}}} \\
& \alpha=(z)=\frac{e^{-a m T_{f}}-e^{-a T_{f}}}{1-e^{-\alpha m T_{f}}}, \beta=1-e^{-a m T_{f}}
\end{aligned}
$$

that can be converted into state space form:

$$
\begin{aligned}
& x_{s y s}[k+1]=A_{s y s} x_{s y s}[k]+B_{s y s} \xi_{s y s}[k] \\
& y_{s y s}[k]=C_{s y s} x_{s y s}[k]
\end{aligned}
$$

\section{FORMULATION OF THE LQG CONTROL PROBLEM FOR} THE ADAPTIVE OPTICS MODEL

The system is assumed to be LTI with state $x[k]$, input $u[k]$, and measurement $y[k]$ given by:

$$
\begin{aligned}
& x[k+1]=A x[k]+B u[k]+G \xi[k] \\
& y[k]=C x[k]+D u[k]+\eta[k]
\end{aligned}
$$

here $\xi[k]$ is the process noise and $\eta[k]$ is the measurement noise. The matrices $A, B$, and $C$ must be augmented in order to account for both the plant dynamics and the output disturbance:

$$
\begin{aligned}
A & =\left[\begin{array}{cc}
A_{s y s} & 0 \\
0 & A_{a t m}
\end{array}\right], B=\left[\begin{array}{c}
B_{s y s} \\
0
\end{array}\right] \\
G & =\left[\begin{array}{c}
0 \\
B_{a t m}
\end{array}\right], C=\left[\begin{array}{ll}
C_{s y s} & C_{a t m}
\end{array}\right], D=0
\end{aligned}
$$


The Strehl ratio is typically used to derive a performance criterion for the controller. The Strehl ratio is the ratio of the peak intensity of a measured point spread function (PSF) to the peak intensity of a perfect diffraction-limited PSF for the same optical system. The Strehl ratio values are from $S=0$ to $S=1$, and the closer $S$ to 1 the better (sharper) the image. The residual wavefront phase (uncompensated disturbance) reduces the Strehl ratio, which can be expressed using the Maréchal approximation[13]:

$$
S_{\text {Marechal }} \approx\left[1-2 \pi^{2}\left(\frac{\Delta \phi_{r}}{\lambda}\right)^{2}\right]^{2}
$$

where $\lambda$ is the observation light's wavelength (m), and $\Delta \phi_{r}$ is the residual wavefront phase (m).

The objective of the controller is to minimise the wavefront phase variance (i.e., keep the wavefront flat), which is equivalent to the maximisation of the Strehl ratio. The cost function for the infinite-horizon control problem can be formulated as:

$$
J=\sum_{k=0}^{\infty}\left(\frac{1}{2} x^{T} Q x+\frac{1}{2} u^{T} R u\right)
$$

The matrix $Q$ can be chosen as $Q=C^{T} C$. The matrix $R$ is set $R=0$ since we do not penalise the control effort.

\section{A. State feedback gain evaluation}

The feedback gain can be found using the solution of the discrete-time algebraic Riccati equations (DARE):

$$
\begin{aligned}
& A^{T} \Omega_{K} A-A^{T} \Omega_{K} B\left(B^{T} \Omega_{K} B+R\right)^{-1} . \\
& \cdot B^{T} \Omega_{K} A+Q=\Omega_{K}
\end{aligned}
$$

The solution of DARE in Eq. Error! Reference source not found. is the matrix $\Omega_{K}$, which is used for the state feedback gain calculation:

$$
K_{\text {gain }}=\left(B^{T} \Omega_{K} B+R\right)^{-1} B^{T} \Omega_{K} A
$$

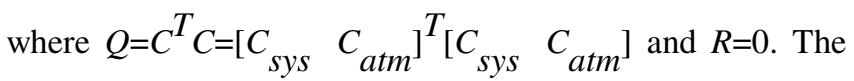
matrix $Q$ contains zeros on the main diagonal. Zeros on the matrix diagonal may cause problems with finite precision computations for the DARE solvers (see Subsection Error! Reference source not found. for the discussion of this issue).

\section{B. State observer gain evaluation}

The observer gain is found from the dual DARE:

$$
\begin{aligned}
& A \Omega_{L} A^{T}-A \Omega_{L} C^{T}\left(C \Omega_{L} C^{T}+\Theta\right)^{-1} . \\
& \cdot C \Omega_{L} A^{T}+V=\Omega_{L}
\end{aligned}
$$

where $V=G G^{T}$ and $G=\left[0, B_{a t m}\right]^{T}$ as indicated in the Eq. Error! Reference source not found.. The choice of $V=G G^{T}$ can lead to the same type of numerical problems as the case of the matrix $Q$ in state feedback case (this issue is discussed in Subsection Error! Reference source not found.).

Using the solution $\Omega_{L}$ of DARE in Eq.Error! Reference source not found., we can evaluate the observer gain:

$$
L_{\text {gain }}=A \Omega_{L} C^{T}\left(C \Omega_{L} C^{T}+W\right)^{-1}
$$

where $W$ is the measurement noise covariance matrix. The feed-through gain $L_{f t}$ can be found in the similar way.

\section{The formulation of the Discrete $L Q G$ controller}

By combining the solutions for the feedback and the observer from the previous subsections, we can formulate the controller as:

$$
\begin{gathered}
u[k]=-K_{\text {gain }}\left(\hat{x}[k]+L_{f t}(y[k]-C \hat{x}[k])\right. \\
\hat{x}[k+1]=A \hat{x}[k]+B u[k]+L_{\text {gain }}(y[k]-C \hat{x}[k])
\end{gathered}
$$

A more convenient form for the implementation is:

$$
\begin{aligned}
& u[k]=-Y \hat{x}[k]+L_{\text {gain }} y[k] \\
& \hat{x}[k+1]=\Psi \hat{x}[k]+\Gamma y[k] \\
& \text { where: } \\
& \mathrm{Y}=K_{\text {gain }}+L_{f t} C \\
& \Psi=A-L_{\text {gain }} C-B\left(K_{\text {gain }}+L_{f t} C\right) \\
& \Gamma=L_{\text {gain }}+B L_{f t}
\end{aligned}
$$

The controller can then be converted to the transfer function representation, which is easier for practical implementation:

$$
C(z)=\mathrm{Y}(z I-\Psi)^{-1} \Gamma+L_{f t}
$$

In the numerical experiments, the controller is used in the form of Eq. Error! Reference source not found.

\section{INITIAL PARAMETERS OF THE NUMERICAL SIMULATIONS}

The goals of the numerical simulations are 1) to establish the correspondence between the noise in the WFS and the measurement noise and 2) evaluate the influence of the measurement noise on the performance of the discrete LQG controller. The cases of bright, medium, and dim guide star correspond to high, medium, and low level photosensor signals, respectively. The variance of the measurement noise is estimated using the optical simulator.

\section{A. Output disturbance (atmospheric turbulence)}

The atmospheric disturbance is simulated as an $\operatorname{AR}(2)$ process. This model describes the atmospheric turbulence with a Kolmogorov-type power spectrum. A white noise process with a standard deviation of $500 \mathrm{~nm}$ was used as an input for the output disturbance.

\section{B. Parameters of the simulated plant}

The model of a Shack-Hartmann WFS with $32 \times 32$ lenslets 
is used in the numerical simulations. The sampling period of the WFS is $T_{f}=10^{-3} \mathrm{sec}$. The time delay introduced by the WFS is equivalent to 1 frame. Such parameters can be considered typical for adaptive optics systems.

The DM was represented by the dynamics of the actuators according to Eq. Error! Reference source not found.:

$$
G_{D M}(s)=\frac{1}{s / a+1}
$$

where the parameter $a$ was $2 * 10^{3}$. In the current simulations, the SISO case of zonal control is considered and the coupling between the actuators in the DM is neglected.

The discrete LQG controller was formulated as described in Section III. The wavefront sensor was assumed to have a delay equal to 0.9 of the sampling time, that is $\tau_{d}=(n-m) \cdot T_{f}$, where $\mathrm{n}=1$ and $\mathrm{m}=0.1$.

\section{DERIVATION OF THE EQUIVALENT MEASUREMENT NOISE} FROM THE PHOTOSENSOR NOISE

The noise is added by the photosensor to the intermediate data that are used for the reconstruction of the signal. The noise is signal-dependent, which means that the amount of noise will vary depending on the ADC and the signal level.

\section{A. The relationship between the photosensor noise and the} measurement noise

The correspondence of the photosensor noise and the measurement noise is difficult to elaborate analytically. This is due to the photosensor noise being added to the intensity image $I$, which is produced by the lenslets of the Shack-Hartman WFS. The intensity image $I$ is used for the calculation of centroid coordinates $\left(x_{k}, y_{k}\right)$ :

$$
x_{k}+N_{1}=\frac{\sum_{i, j} x_{i, j}\left(I_{i, j}+N\right)}{\sum_{i, j}\left(I_{i, j}+N\right)}
$$

where $N$ is the photosensor noise; $N_{1}$ is the centroid noise due to the noise in the intensity image $I$ that is used for the calculation of turbulent and reference centroid coordinates. Therefore, one needs to simulate the complete acquisition and reconstruction process in order to evaluate the measurement noise levels.

\section{$B$. The procedure for evaluation of measurement noise}

Many sources of noise contribute to the resulting image produced by photosensors. A simple noise model[14] can be established using photo response non-uniformity (PRNU), photon shot noise, read noise, dark current Fixed Pattern Noise and dark current shot noise. However, other noise sources must be considered to form a more comprehensive model. The comprehensive model of the CCD photosensor[8] was used to obtain the different noise levels in the signal from the photo- sensor.

We have considered the cases of bright, medium, and dim guidestars. The light noise, namely photon shot noise and photo-response non-uniformity (PRNU), was considered. The equivalent measurement noise was evaluated for the case of either 8 or 10 bit photosensor ADC's in the WFS.

The noise was generated as follows. First, one layer of turbulence with Kolmogorov spectrum was generated and sensed with the model of the WFS; no noise was added by the photosensor model at this time. Next, the same wavefront was sensed with the model of a photosensor that added the photon shot noise and the PRNU noise. After each "acquisition" by the model of the photosensor, the numerical model of the ADC performed the quantisation at either 8 or 10 bit. Then the two wavefronts were subtracted from each other, and the standard deviation of the remainder signal was calculated. Such a procedure was repeated 32 times in order to obtain an averaged noise level.

\section{Results on the evaluation of equivalent measurement} noise

The results for the measurement noise in cases of either 8 or 10 bit photosensor ADC are summarised in Fig. 1. One can see that the measurement noise levels are similar in both cases. In the absence of the measurement noise, the residual signal (residual wavefront) is estimated as $46.2 \mathrm{~nm}$ in the case of 2 frames delays and a turbulence standard deviation of $500 \mathrm{~nm}$.

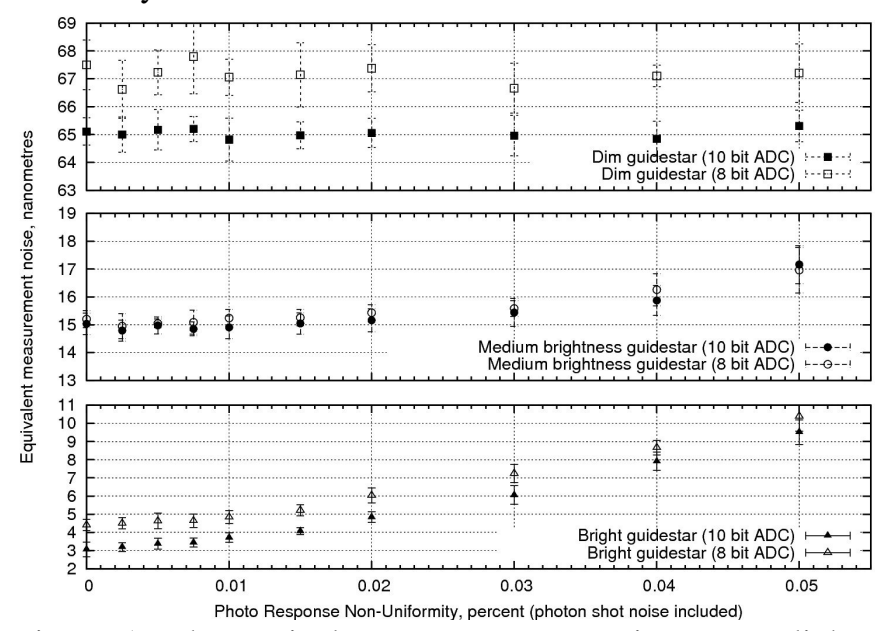

Figure 1: The equivalent measurement noise versus light noise level for the case of 8 and 10 bit ADC in the wavefront sensor. 


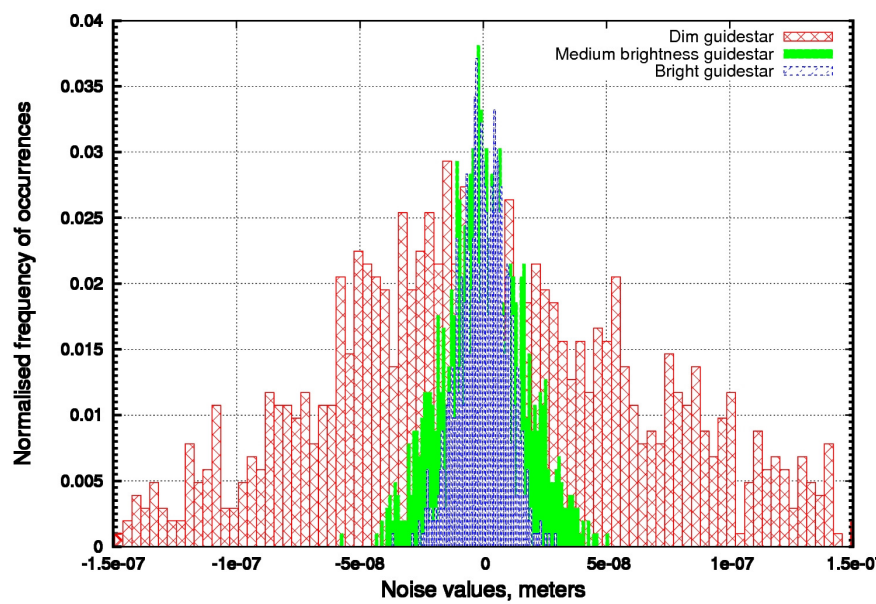

Figure 2: Probability density function of the measurement noise for the case of photon shot noise and PRNU 5\% (worst case scenario).

The probability density of the measurement noise, which is similar for both the 8 and 10 bit case, is shown in Fig. 2. These noise parameters are used for the evaluation of the influence of the measurement noise on the discrete LQG controller performance in further Subsection Error! Reference source not found.

VI. RESULTS ON SIMULATION OF THE MEASUREMENT NOISE INFLUENCE ON THE PERFORMANCE OF A DISCRETE LQG CONTROLLER

The equivalent measurement noise values were used for the numerical simulations in order to establish the impact on the residual signal in the LQG control system. The cases of 8 and 10 bit ADC's of the WFS photosensor were considered as these are the usual choices in the design of a modern WFS. The duration of the simulation was set to 3 seconds, with 3000 data points collected for each run (16 runs were performed for each parameter set).

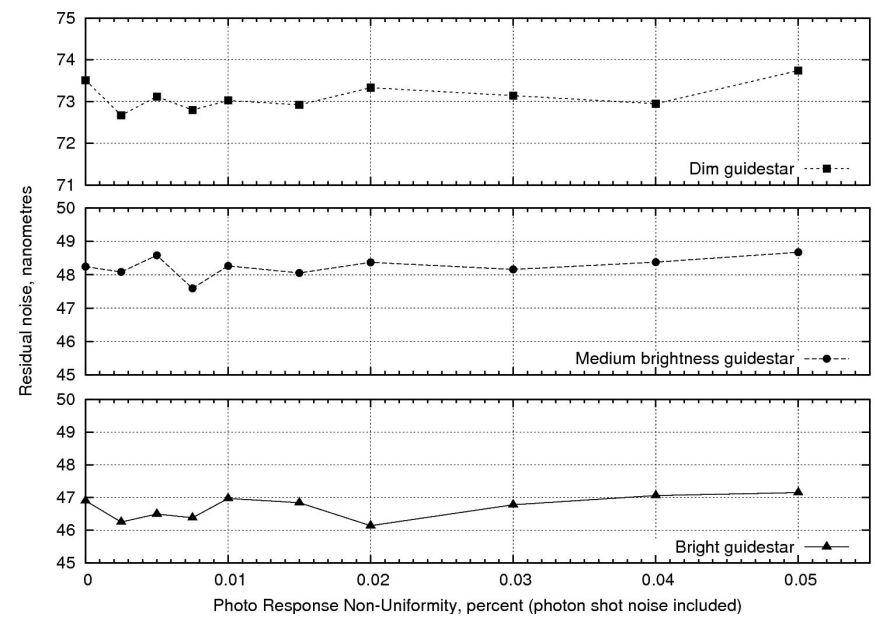

Figure 3: The performance of the discrete LQG controller in the presence of the measurement noise: the residual signal (uncompensated atmospheric turbulence) level versus the noise in the photosensor noise.
The residual noise value, which is the standard deviation of the residual signal, is shown in Fig. 3. The probability density for the residual noise is provided in Fig. 4. These results can be interpreted as the remaining uncompensated atmospheric turbulence (output disturbance) that degrades the astronomical images. Ideally, the residual level of the signal should be zero; however, even in the absence of measurement noise, the residual signal (residual wavefront) is estimated as $46 \mathrm{~nm}$ due to the 2 frame delay in the system.

The results in Fig. 3 can be represented using the Strehl ratio from Eq. Error! Reference source not found.. As above, even in the absence of noise, the residual signal is about $46 \mathrm{~nm}$ which gives a Strehl ratio $S=0.69$. In the presence of measurement noise, the residual signal will increase by the values that are presented in Fig. 1 (these values correspond to the photosensor noise and do not include the noise due to time delays).

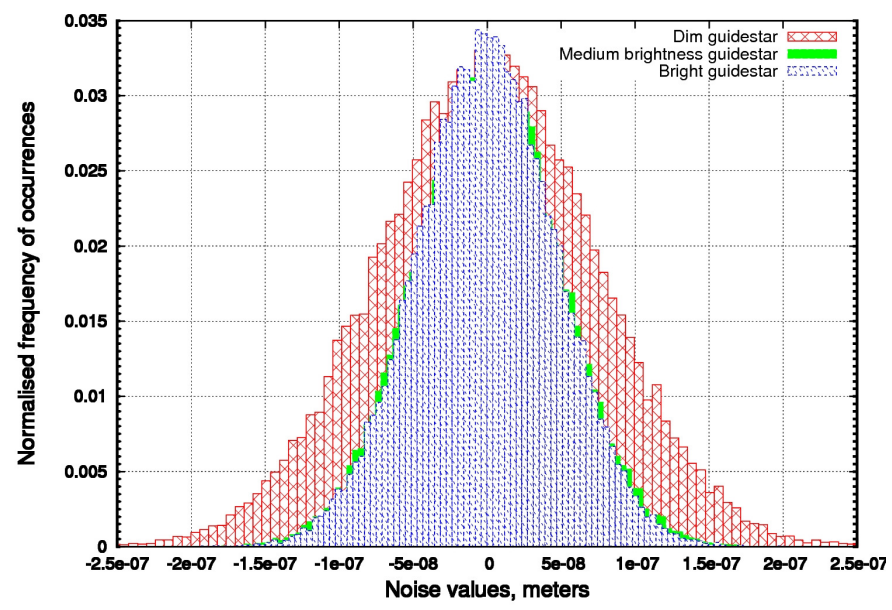

Figure 4: Probability density function of the residual noise for the case of CCD photosensor with 10 bit ADC, photon shot noise and PRNU 5\% (worst case scenario).

The LQG controller reduces the noise to the levels, presented in Fig. 3. The results are summarised in Table 1 in the form of the noise values and the corresponding Strehl ratio. The results summarised in Table 1 show that the LQG controller considerably attenuates the measurement noise, even in the case of relatively strong noise (photon shot noise and 5\% PRNU noise).

Table 1: The performance of the discrete LQG controller: measurement noise influence on the Strehl ratio

\begin{tabular}{lllll}
\hline $\begin{array}{l}\text { Noise } \\
\text { type }\end{array}$ & Open-loop & \multicolumn{3}{c}{$\begin{array}{l}\text { Closed-loop } \\
\text { (LQG) }\end{array}$} \\
\hline & $\begin{array}{l}\text { equivalent } \\
\text { noise, nm }\end{array}$ & $\begin{array}{l}\text { Strehl } \\
\text { ratio }\end{array}$ & $\begin{array}{l}\text { Residual } \\
\text { signal, nm }\end{array}$ & $\begin{array}{l}\text { Strehl } \\
\text { ratio }\end{array}$ \\
\hline Bright & & guidestar $\square \square$ shot
\end{tabular}

noise

$\begin{array}{lllll}\text { shot noise } & 49.5 & 0.65 & 46.9 & 0.68\end{array}$




\begin{tabular}{|c|c|c|c|c|}
\hline shot + PRNU & 55.6 & 0.57 & 47.1 & 0.68 \\
\hline $\begin{array}{l}\text { Medium } \\
\text { noise }\end{array}$ & brightness & \multicolumn{3}{|c|}{ guidestar $\square \square \square$ shot } \\
\hline shot noise & 61.3 & 0.49 & 48.2 & 0.66 \\
\hline shot + PRNU & 63.3 & 0.46 & 48.7 & 0.66 \\
\hline $\begin{array}{l}\text { Dim } \\
\text { noise }\end{array}$ & & \multicolumn{3}{|c|}{ guidestar $\square \square \square$ shot } \\
\hline shot noise & 111.3 & 0.01 & 73.5 & 0.33 \\
\hline shot + PRNU & 111.5 & 0.01 & 73.9 & 0.32 \\
\hline
\end{tabular}

\section{RESULTS DISCUSSION}

The results on the performance of the LQG controller in this paper are presented for the decoupled system, that is, for the model of a deformable mirror (DM) where the influence of actuators on their neighbours is neglected. However, in a real continuous faceplate DM, the coupling can be considerable. The appropriateness of the decoupled model is discussed further with the reformulation of the Linear Quadratic Gaussian (also called Infinite Horizon) controller in the Receding Horizon Control fashion.

\section{A. Connection between Infinite and Receding Horizon Con-} trol

The minimisation of the cost function (performance index) from Eq. Error! Reference source not found. can be performed through Riccati equations as well as using the Receding Horizon Control formalism[15]. The cost function is:

$$
J=\frac{1}{2} x^{T} C^{T} C x+\frac{1}{2} X^{T} Q X+\frac{1}{2} U^{T} R U
$$

where $N_{p}$ is a state prediction horizon, and $N_{c}$ is a control prediction horizon.

We define matrices $\Gamma$ and $\Omega$ as:

$$
\Gamma=\left[\begin{array}{ccc}
B & \cdots & 0 \\
A B & \cdots & 0 \\
\vdots & \ddots & \vdots \\
A^{N_{p}-1} B & \cdots & A^{N_{p}-N_{c}} B
\end{array}\right], \Omega=\left[\begin{array}{c}
A \\
A^{2} \\
\vdots \\
A^{N_{p}}
\end{array}\right]
$$

The state vector can be expressed as:

$$
X=\Gamma U+\Omega x
$$

Substituting (Error! Reference source not found.) into the cost function (Error! Reference source not found.) we obtain:

$$
J=\bar{V}+\frac{1}{2} U^{T} H U+U^{T} F x
$$

where the term $\bar{V}$ is independent of $U$. The Hessian matrix
$H$ and the matrix $F$ are defined as:

$$
H=\Gamma^{T} Q \Gamma+R, F=\Gamma^{T} Q \Omega
$$

In the unconstrained case, the cost function in (Error! Reference source not found.) is minimised when:

$$
U^{o p t}=-H^{-1} F x=-K_{M P C \cdot \text { gain }} x
$$

where $x$ is typically replaced by the vector of estimated states obtained by a Kalman filter.

It is easy to verify [16] that the eigenvalues of the discretetime MPC system are almost identical to those obtained from discrete LQR system.

B. Validity of the decoupled model in case of coupling between nearest neighbour actuators in deformable mirror

In the case when there is no coupling between actuators, the Hessian of the decoupled problem is diagonal and therefore very sparse (98\% of elements are zeros). The matrix is normalised for illustrative purpose, and the zero elements are represented in white to stress the sparse structure. The coupling between the nearest actuators makes the dynamics more complex, which is reflected in the structure of the Hessian matrix.

The validity of decoupled control in the case of actuator coupling can be assessed by thresholding the Hessian matrix of the coupled model. We use the thresholded Hessian matrix to generate control signals for the coupled (un-thresholded) plant model (i.e., coupled actuators of the deformable mirror).

We measured the standard deviation of the residual uncompensated disturbance as a function of the threshold value to find a suitable threshold level. The value of the threshold was calculated as $\tau=\max (\operatorname{diag}(H)) * 10^{-n}$, where $H$ is the Hessian matrix. The elements of matrix $X$ that was less than the threshold was zeroed.

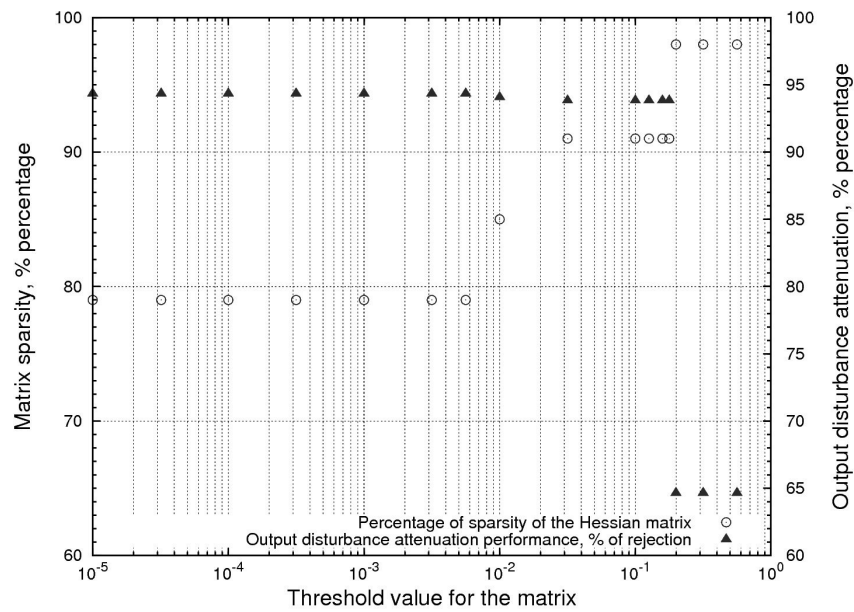

Figure 5: The influence of the threshold level on sparsity of the Hessian matrix $H$ and disturbance rejection performance.

Application of a threshold up to 
$\tau_{H . \text { safe }}=\max (\operatorname{diag}(H)) * 10^{-1}$ increases the sparsity of the

Hessian from $79 \%$ to $92 \%$. Such a threshold does not influence the disturbance rejection performance: the output disturbance is rejected by $94 \%$.

However, starting from the threshold $\tau_{H \text {.unsafe }}=\max (\operatorname{diag}(H)) * 10^{-0.9}$, the performance of the RHC controller drops from $94 \%$ to $65 \%$. This is because we try to control a coupled plant assuming a decoupled (excessively thresholded) model[17]. Excessive thresholding of matrices brings them closer to the decoupled case that leads to mismodelling (i.e., the coupled plant is controlled assuming a decoupled model). Such a mismodelling causes inferior performance of the RHC controller. That is, although the decoupled model can be used to control the coupled plant, the disturbance rejection performance of the controller will be inferior. The decoupled model is used in the simulations to study the effects of the realistic measurement noise and the model mismatch separately.

VIII. NUMERICAL STABILITY OF THE OPTIMAL CONTROL FOR ADAPTIVE OPTICS SYSTEMS

A typical deformable mirror for the adaptive optics system may consist of thousands of actuators, and therefore the matrices of the LQG controller can be very large. Although most of the calculations are performed off-line, the evaluation of the control matrices can be difficult due to the finite precision of the computations.

Consider the effect of a sensor delay. The state space model of a two cycle delay[12] in output $y$ is the following:

$$
\begin{aligned}
& x_{\text {aug }}[k+1]=A_{\text {aug }} x_{\text {aug }}[k]+B_{\text {aug }} u[k] \\
& y_{d}[k]=C_{\text {aug }} x_{\text {aug }}[k]
\end{aligned}
$$

where the augmented matrices are:

$$
\begin{gathered}
x_{\text {aug }}[k+1]=\left[\begin{array}{c}
x[k+1] \\
y_{1 d}[k+1] \\
y_{2 d}[k+1]
\end{array}\right], A_{\text {aug }}=\left[\begin{array}{lll}
A & 0 & 0 \\
C & 0 & 0 \\
0 & I & 0
\end{array}\right] \\
B_{\text {aug }}=\left[\begin{array}{c}
B \\
0 \\
0
\end{array}\right], C_{\text {aug }}=\left[\begin{array}{lll}
0 & 0 & 1
\end{array}\right]
\end{gathered}
$$

Here $y_{d}$ is the output $y$ that is delayed by two cycles.

Due to augmentation of the state matrices, there may be numerical problems in solving of the corresponding DARE for the LQR. This issue is considered in the subsections below.

\section{A. Numerical stability of the Riccati equations for Infinite} Horizon Control

In the standard LQR formulation, the weight matrix
$Q=C^{T} C=\left[\begin{array}{lll}C_{s y s} & C_{a t m}\end{array}\right]^{T}\left[\begin{array}{ll}C_{s y s} & C_{a t m}\end{array}\right]$, which is used in the cost function in Eq.Error! Reference source not found., may loose the positive semi-definiteness and become illconditioned. This may cause problems with finite precision computations for the DARE solvers. The matrix elements that correspond to the output disturbance are small compared to the plant elements (see Fig. 6). The determinant of the matrix $Q$ for the solution of Riccati equation can be close to zero and in turn leads to numerical problems in solving the Riccati equations.

Consider the singular value decomposition (SVD) of the matrix $Q$. Define the condition number[18] as $\kappa(Q)=\sigma_{\max } / \sigma_{\min }$. Even in case of no time delays, the condition number for the matrix $Q$ is of order $\kappa(Q) \approx 5 \cdot 10^{15}$ for the $5 \times 5$ actuator model (this value is obtained with diagonal loading of 1 eps). This is not surprise since the matrix $Q$ contains many zeros (or very small numbers) on the main diagonal.

Similar problem occurs with the $V=G G^{T}$, where $G=\left[0, B_{a t m}\right]^{T}$. The matrix $V$ is used for the calculation of steady-state Kalman estimator. The condition number for the matrix $V$ is of order $\kappa(Q) \approx 5 \cdot 10^{9}$ for the $5 \times 5$ actuator model (see Fig. 7). As before, the value is obtained with diagonal loading of 1eps, otherwise $\kappa(V)=+\infty$ since the smallest eigenvalue is zero.

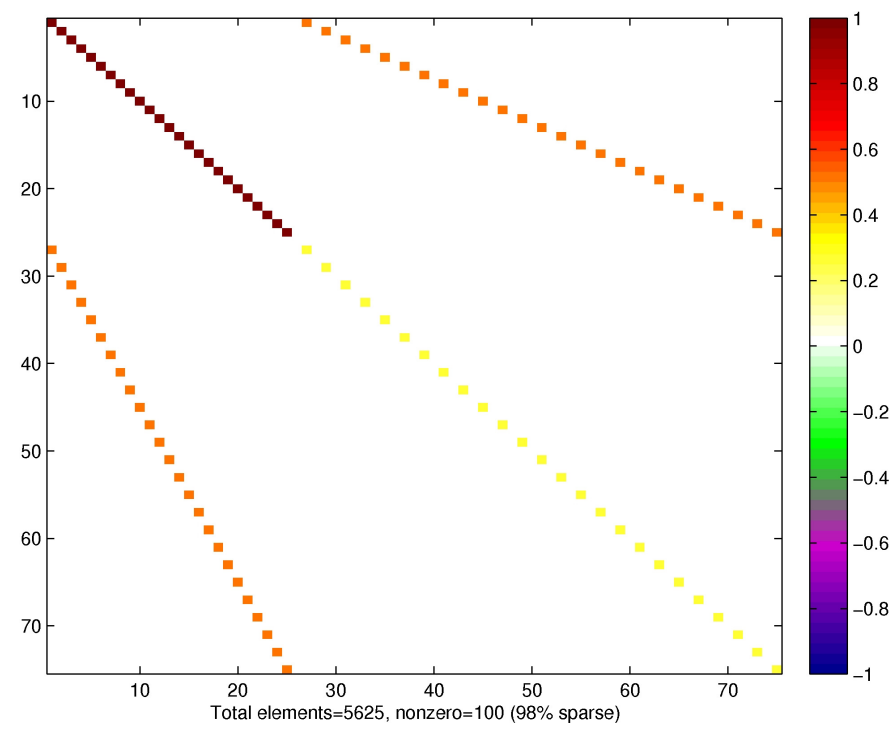

Figure 6: The structure of the matrix $Q$ for the $\mathrm{LQR}$ (the matrix is normalised for illustrative purposes. 


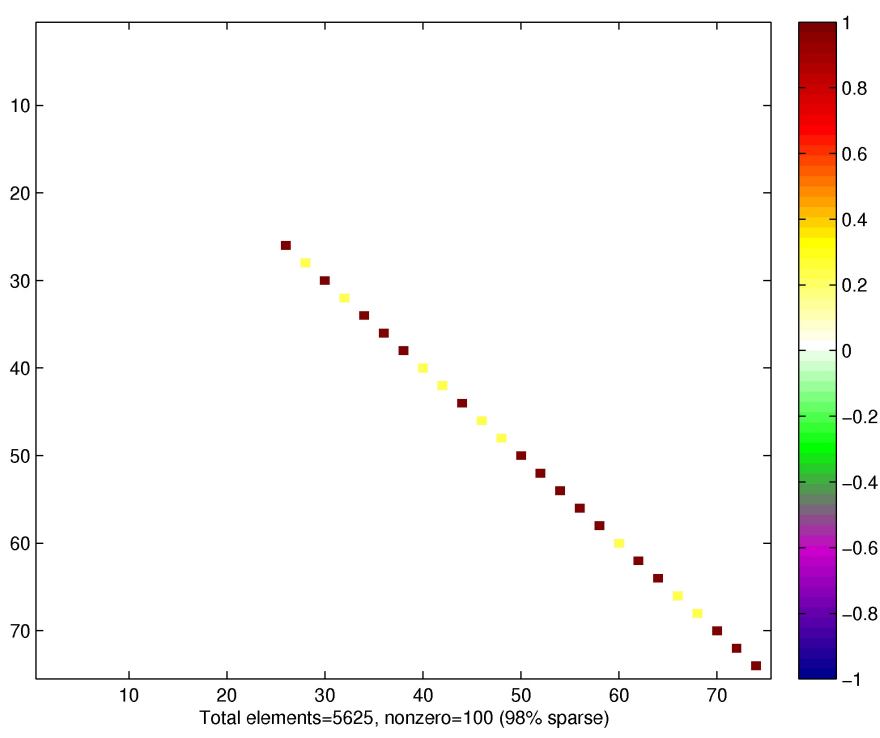

Figure 7: The structure of the matrix $V=G G^{T}$ for the LQR (the matrix is normalised for illustrative purposes.

The algorithm that is implemented in the MATLAB's DARE routine uses the QZ algorithm[19] to deflate the extended symplectic pencil and compute its stable invariant subspace. In case of poor conditioning, as described above, the algorithm often terminates with error: evaluation of eigenvalues is numerically unstable because the spectrum is too near the imaginary axis.

Augmentation of the state matrices (see Eq. Error! Reference source not found.) for accounting the delays of two cycles (typical for adaptive optics system) does not noticeable change the numerical properties of the matrices $Q_{a u g}$ and $V$. The problems with the conditioning remain the same: $\kappa\left(Q_{\text {aug }}\right) \approx 1 \cdot 10^{9}$ and $\kappa(V) \approx 1 \cdot 10^{9}$.

\section{A. Circumventing the numerical stability problem in case of $L Q R$}

Since the condition number $\kappa(Q)$ can be large, indicating possible numerical problems, the regularisation of the weighting matrix $Q$ is therefore necessary. One can use the $d i$ agonal loading technique for the regularisation that is analogous to Tikhonov regularisation[20]:

$$
Q=Q+\delta I
$$

where $\delta$ is the regularisation term, and $I$ is an identity matrix. By increasing the regularisation term in Eq. Error! Reference source not found. to $10^{7}$, the condition number $\kappa(Q)$ has been reduced to $10^{5}$. However, by that point the calculated LQG controller becomes unstable.

To summarise, we can say that the numerical properties of the augmented matrices $Q$ and $V$, which are used for state feedback and state estimator respectively, are prone to numerical problems. Although the numerical problems can be alleviated (e.g., diagonal loading), a better solution would be to use alternative formulation of the optimal control. The most attractive technique for the optimal control formulation is Receding Horizon Control formalism.

B. Numerical stability of matrices in the Receding Horizon Control formulation

Receding Horizon Control (RHC) in the standard formulation uses two composite matrices to solve the quadratic optimisation problem, namely $H=\Gamma^{T} Q \Gamma+R \quad$ and $F=\Gamma^{T} Q \Omega$. Therefore, the numerical stability of the solution for the optimal control will depend on the numerical properties of those matrices.

In the case when no augmentation is performed for the time delays (undelayed case), the condition numbers for the are Hessian matrix $H$ and $F$-matrix are $\kappa(H)=1.3$ and $\kappa(F)=1.3$ for the case of $5 \times 5$ actuators. This is a considerable improvement in the numerical properties of the matrices over the conventional LQR formulation (as seen in the subsection above). The structure of the matrices is shown in Fig. 8 and Fig.9 for the Hessian and F matrix respectively.

The augmentation of the RHC matrices has been performed in the similar way as LQR matrices. The size of the $F$ matrix enlarged accordingly, while the size of the Hessian remains the same. More important is that the condition number of augmented matrices does not change; that is, the optimization problem remains strictly convex. This makes the formulation of the RHC preferable over the LQR for practical implementation of the optimal controllers in adaptive optics, particularly in a sense of numerical computations.

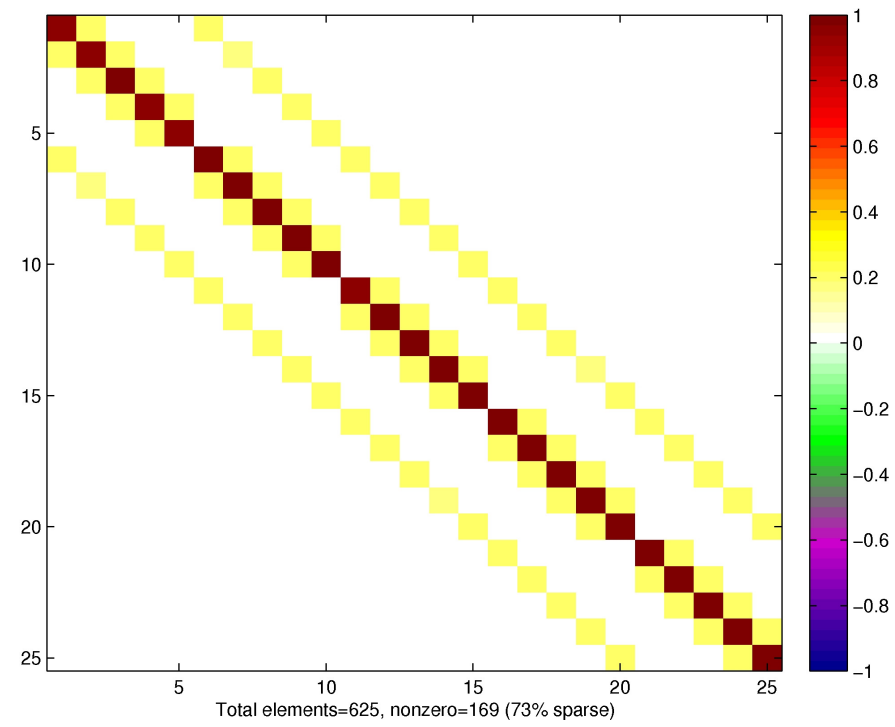

Figure 8: The structure of the Hessian matrix $H$ for the RHC. 


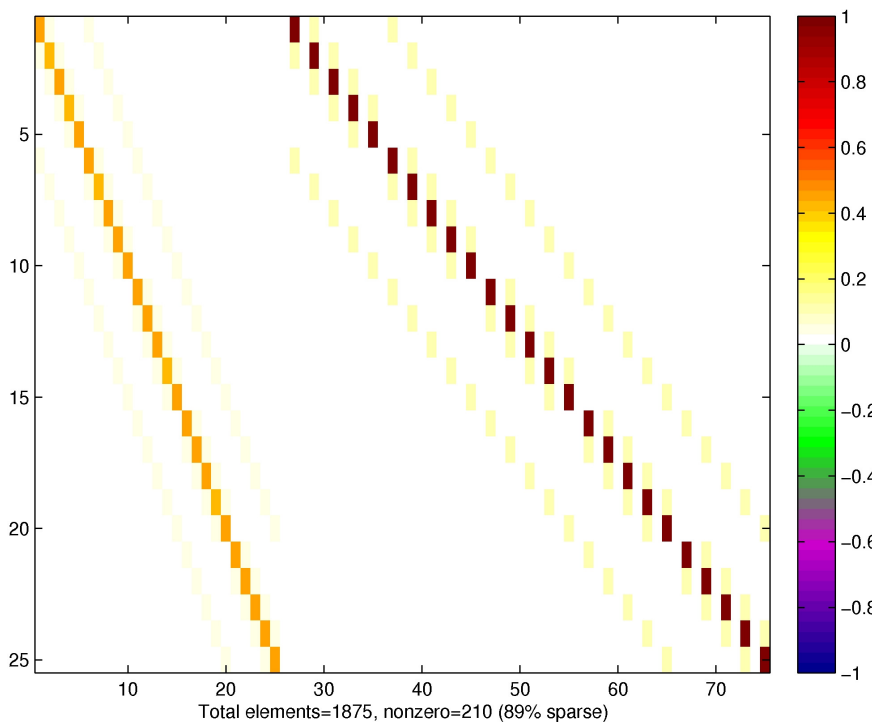

Figure 9: The structure of the matrix $F$ for the RHC.

The observed numerical properties of the RHC matrices suggest that the problem is strongly convex and therefore the QP algorithms will guarantee the convergence to a unique solution[17].

\section{CONCLUSIONS}

In this paper we presented the results of numerical simulations for the optimal control of an adaptive optics system in the presence of realistic measurement noise. We used a previously developed comprehensive model of the CCD photosensor to establish the correspondence between the noise in the wavefront sensor and the measurement noise in the control system. The influence of the noise on the performance of the LQG controller for the adaptive optics system was considered. The article also provides a discussion on the numerical stability of the discrete controller.

The performance of the formulated LQG controller was estimated for the case of dim, medium and bright guidestars. According to the results of the numerical simulations, the equivalent noise levels for 8 and 10 bit photosensor ADC are very similar. However, the impact of the photo-response nonuniformity of the photosensor was expected to be more significant: the Strehl ratio was about 0.66 (out of 1 ). The performance of the LQG controller in case of strong measurement noise was expected to be worse, yet the Strehl ratio was estimated as 0.33 . This can be considered as reasonably good performance, especially as the measurement noise is very large.

From the implementation perspective, we found that the Receding Horizon Control (RHC) formulation is less prone to numerical problems than the classical LQR formulation. The Hessian matrix is very well conditioned and sparse, which can be exploited in many quadratic optimisation algorithms.

The numerical simulations show promising results of the performance of the discrete LQG controller even in case of a strong measurement noise. The RHC formulation of the control problem seems to be preferable due to better numerical stability of the resulting matrices.

\section{REFERENCES}

[1]E. Gendron and P. Léna, "Astronomical adaptive optics. II. Experimental results of an optimized modal control." Astronomy and Astrophysics Supplement Series, vol. 111, p. 153, 1995.

[2]C. Dessenne, P. Madec, and G. Rousset, "Optimization of a predictive controller for closed-loop adaptive optics," Applied optics, vol. 37, no. 21, pp. 4623-4633, 1998.

[3]R. N. Paschall and D. J. Anderson, "Linear quadratic gaussian control of a deformable mirror adaptive optics system with time-delayed measurements," Appl. Opt., vol. 32, no. 31, pp. 6347-6358, Nov 1993.

[4]B. Le Roux, J. Conan, C. Kulcsár, H. Raynaud, L. Mugnier, and T. Fusco, "Optimal control law for classical and multiconjugate adaptive optics," Journal of the Optical Society of America A, vol. 21, no. 7, pp. 1261-1276, 2004. [5]C. Petit, J.-M. Conan, C. Kulcsár, H.-F. Raynaud, T. Fusco, J. Montri, and D. Rabaud, "Optimal control for multi-conjugate adaptive optics," Comptes Rendus Physique, vol. 6, no. 10, pp. 1059 - 1069, 2005.

[6]K. Hinnen, M. Verhaegen, and N. Doelman, "A data-driven H2-optimal control approach for adaptive optics," IEEE Transactions on Control Systems Technology, vol. 16, no. 3, pp. 381-395, 2008.

[7]C. Petit, J. Conan, C. Kulcsár, H. Raynaud, T. Fusco, J. Montri,

F. Chemla, and D. Rabaud, "Off-axis adaptive optics with optimal control: experimental and numerical validation," in Proceedings of SPIE, volume 5903, pages 59030P, 2005.

[8]M. V. Konnik and J. Welsh, "On numerical simulation of high-speed ccd/cmos-based wavefront sensors in adaptive optics," in Proceedings of SPIE Optical Engineering + Applications, San Diego, CA, paper 8149-15, 2011.

[9]A. Wirth, J. Navetta, D. Looze, S. Hippler, A. Glindemann, and

D. Hamilton, "Real-time modal control implementation for adaptive optics," Applied optics, vol. 37, no. 21, pp. 4586-4597, 1998.

[10] C. Petit, S. Meimon, T. Fusco, C. Kulcsár, and H.-F. Raynaud, "Hybrid LQG/integrator control for the VLT eXtreme AO system SPHERE," in IEEE International Conference on Control Applications, Yokohama, Japan, September 8-10, 2010.

[11] U. Frisch, Turbulence: the legacy of A.N. Kolmogorov. Cambridge University Press, 1995.

[12] G. Franklin, D. Powell, and M. Workman, Digital control of dynamic systems. Addison-Wesley; 3rd edition, 1997.

[13] A. Maréchal, "Study of the combined effects of diffraction and geometrical aberrations on the image of a luminous point," Rev d'Optique, vol. 26, pp. 257-277, 1947.

[14] Gerald Holst, CCD arrays, cameras, and displays.JCD Publishing, 1998.

[15] Graham Clifford Goodwin, Maria Marta Seron, and José De Doná, Constrained Control and Estimation: An Optimisation Approach. Springer, Berlin, 2005.

[16] L. Wang, Model predictive control system design and implementation using MATLAB. Springer Verlag, 2009.

[17] Mikhail V. Konnik, José De Doná, and James Stuart Welsh, "On application of constrained receding horizon control in astronomical adaptive optics," in Proceedings of SPIE Astronomical Telescopes + Instrumentation Adaptive Optics Systems III, vol. 8447-110, 2012.

[18] G. Stewart, Introduction to matrix computations. $1 \mathrm{em}$ plus $0.5 \mathrm{em}$ minus 0.4emAcademic press New York, 1973.

[19] I. Arnold, W.F. and A. Laub, "Generalized eigenproblem algorithms and software for algebraic riccati equations," Proceedings of the IEEE, vol. 72, no. 12, pp. 1746 - 1754, dec. 1984.

[20] C. Lawson and R. Hanson, Solving least squares problems., Society for Industrial and Applied Mathematics, 1995. 


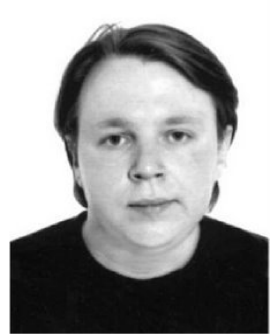

Mikhail V. Konnik was born in Moscow, Russia, in 1982. He received the M.S. degree in Physics from Moscow Engineering Physics Institute (MEPhI), Russia, in 2006. From 2006 to 2010 he was a Senior Engineer in Laser Physics Department, MEPhI, Russia. His research interests include optical-digital systems and control problems in adaptive optics.

$\mathrm{He}$ is currently working towards the Ph.D. degree in Electrical Engineering in the University of Newcastle, Australia.

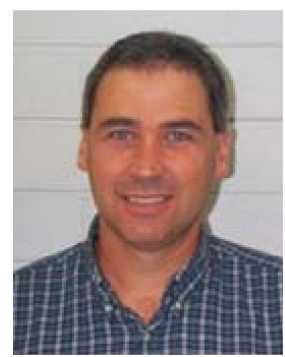

James S. Welsh was born in Maitland, Australia, in 1965. He received the B.E. degree (Hons. I) in electrical engineering from The University of

Newcastle, Callaghan, NSW, Australia, in 1997. Dr. Welsh received his $\mathrm{PhD}$ which focused on illconditioning problems arising in system identification in 2004 from the same university.

During the last several years, he has been actively involved in research projects which include, Powertrain Control, Model Predictive Control and System Identification Toolboxes, with the Centre for Complex Dynamic Systems and Control, the University of Newcastle. His research interests include auto-tuning, system identification, and process control. Dr Welsh is currently employed as a Senior Lecturer in the School of Electrical Engineering and Computer Science at the University of Newcastle. 